\title{
Heterogenous Morphologic Forms of Goiter in Autoimmune Thyroid Disease: An Insight based on a Prospective Surgical Series of 88 Cases
}

\author{
PRK Bhargav
}

\begin{abstract}
Two commonest forms of autoimmune thyroid disease (AITD) are Graves' disease (GD) and Hashimoto's thyroiditis (HT) with a diffuse goiter. The nature of goiter apart from clinical presentation is crucial in the management of AITD. But, the goiter is not always diffuse, leading to diagnostic confusion. In this context, we conducted a prospective study on the goiter morphology in AITD. This is a prospective study conducted in Endocrine Surgery department of a teritiary care teaching hospital in South India over a period of 1 year. The cohort is a surgical series of 88 cases of AITD (GD = 53; HT = 35). Morphology of all the ex vivo specimens were studied, documented and correlated with clinical and radiological forms of goiter. Sex ratio was $M: F=74: 14$. Mean age for $\mathrm{GD}=30.7$ years $(17-46)$ and $\mathrm{HT}=38.2$ years $(31-52)$. In $\mathrm{GD}$, the morphology was diffuse $=34$; Unilateral hyperplasia $(\mathrm{ULH})=9$; atrophic $=4 ;$ nodular $=5$ and Marine Lenhart syndrome $=1$. $\ln \mathrm{HT}$, diffuse $=16 ; \mathrm{ULH}=10$ and nodular goiter $=9$. The correlation between the radiology and goitrous morphology was statistically significant and more concordant than clinico-morphological correlation. Autoimmune thyroid disease has heterogenous goitrous forms. Macroscopic morphological evaluation of goiter complements the clinicoradiological-pathological diagnosis of AITD leading to optimal diagnosis, counseling, follow-up.
\end{abstract}

Keywords: Graves' disease, Hashimoto's thyroiditis, Autoimmune disease, Morphology, Goiter.

How to cite this article: Bhargav PRK. Heterogenous Morphologic Forms of Goiter in Autoimmune Thyroid Disease: An Insight based on a Prospective Surgical Series of 88 Cases. World J Endoc Surg 2014;6(2):71-76.

Source of support: Nil

Conflict of interest: None

\section{INTRODUCTION}

Graves' disease (GD) and Hashimoto's thyroiditis (HT) are two of the most frequent thyroid disorders in the communities, Worldwide. ${ }^{1-5}$ Both of them are organ specific autoimmune thyroid disease (AITD). ${ }^{6}$ One of the important clues in the definitive diagnosis of AITD is the goiter morphology.

\footnotetext{
Consultant Endocrine Surgeon

Department of Endocrine and Metabolic Surgery, Mamata Medical College and Superspeciality Hospital, Khammam Andhra Pradesh, India

Corresponding Author: PRK Bhargav, Consultant Endocrine Surgeon, Department of Endocrine and Metabolic Surgery Mamata Medical College and Superspeciality Hospital Khammam, Andhra Pradesh, India, Phone: 08666617633 e-mail: kingbhargav@gmail.com
}

Usually, both GD and HT have diffuse goiter due to bilateral symmetrical involvement of thyroid gland by the disease process. ${ }^{7}$ But, in 20 to $30 \%$ of cases, they may be associated with nodules or assymetrical enlargement. ${ }^{8-11}$ The variability in proportion of nodularity depends upon clinical or sonographic methods of evaluation. In a classical case of AITD (i.e. with usual clinical presentation, cardinal signs and diffuse goiter), the standard diagnostic protocol with imaging and serology suffices, but appears to be insufficient in AITD with atypical clinical picture and nodular goiters. This leads to diagnostic confusion in cases with atypical clinical picture. Scanty data exists on the goiter morphology in AITD. To address this issue, we conducted a prospective study on goiter morphology in AITD with specific emphasis on its clinical impact in the management of AITD.

\section{MATERIALS AND METHODS}

This is a prospective observational study conducted in the Endocrine Surgery department of a tertiary care teaching hospital in Southern India. This study included 88 cases of AITD, treated surgically between October 2010 to September 2011 (12 months). Informed consent was obtained from all the subjects. The study complied with the international ethical norms according to Helsinki Declaration-Ethical Principles for Medical Research Involving Human Subjects. ${ }^{12}$ All these cases were clinically and biochemically diagnosed. Clinical and radiological features were documented. Inclusion criteria was surgically treated cases of AITD with histopathological confirmation. Exclusion criteria was cases with malignant nodules in AITD as confirmed on ultrasound guided cytopathology for papillary thyroid cancer and on histopathology for follicular cancer. Indications for surgery in GD were large goiters, drug intolerant or failed or relapsed cases and those with associated exophthalmos. Surgical indications in HT were large goiter wit compressive symptoms, recurrent painful thyroiditis and persistent goiter inspite of thyroxine replacement for more than 2 years.

Morphology of all the excised fresh thyroidectomy specimen were studied within the operation theater by the operating surgeon. Macroscopic evaluation of both gross uncut 
and bisected specimen was done by inspection, palpation and were photographed. The findings including measurements were recorded in a proforma for each patient. The morphology of ex vivo goiter specimen was compared with clinical and radiological information. The morphological parameters examined were: (A) Goiter surface-Diffuse or nodular; (B) weight of the ex vivo thyroidectomy specimen; (C) Number of nodules-none, solitary or multiple; (D) Demarcation between nodule margin and surrounding parenchyma. The goiters were bisected in a single plane across both lobes and isthmus. Statistical analysis was performed with SPSS software 12.0. Descriptive statistics, chi-square test with yates correction and Fisher's exact test were utilized to analyze the data. $p$-value of $<0.05$ was considered statistically significant.

\section{RESULTS}

The cohort included 88 cases of AITD-Graves' disease $(\mathrm{n}=53)$ and Hashimoto's thyroiditis $(\mathrm{n}=35)$. Female to male proportion was $74: 14$. In the cohort, $84 / 88$ cases underwent Total thyroidectomy. For GD, the indications for surgery were poor drug compliance, complications of antithyroid drugs, large goiter and cosmetic reasons. For HT, indications were large goiter with pressure effects, persistent goiter, fear of malignancy and painful thyroiditis. The commonest histopathology was hyperplastic goiter in $60 \%$ of GD and diffuse HT in $76 \%$ of HT cases. The histopathological details are tabulated in Table 1. Frequency distribution of major

Table 1: Histopathology of thyroid specimen in autoimmune thyroid disease

\begin{tabular}{ll}
\hline Graves' disease (GD) & Hashimoto's thyroiditis $(H T)$ \\
\hline Hyperplastic goiter $=32$ & HT $=29$ \\
Colloid goiter = 10 & HT with colloid nodule = 4 \\
Adenomatous hyperplasia = 5 & HT with follicular adenoma = 2 \\
Hyperplastic goiter + & - \\
Hashimoto's disease $=6$ & \\
\hline
\end{tabular}

Table 2: Major clinical categories of goiter in this study

\begin{tabular}{lll}
\hline $\begin{array}{l}\text { Morphology of } \\
\text { goiter }\end{array}$ & $\begin{array}{l}\text { Graves' disease } \\
(\mathrm{GD}) \mathrm{N}=53\end{array}$ & $\begin{array}{l}\text { Hashimoto's thyroiditis } \\
(\mathrm{HT}) \mathrm{N}=35\end{array}$ \\
\hline Diffuse & 31 & 22 \\
Nodular & 18 & 13 \\
Nonpalpable & 4 & - \\
\hline
\end{tabular}

clinical goiter forms is depicted in Table 2. 58.5 and $62 \%$ were clinically diffuse goiters in GD and HT respectively.

Detailed frequency distribution of morphological, clinical and radiological forms of goiter in both GD and HT is shown in Table 3. The correlation between clinico-radiological and clinicomorphological goitrous forms for GD is graphically shown in Table 4. The correlation between morphology and clinical forms of goiter was statistically significant, suggestive of discordant findings. This is especially evident in nodular and diffuse goiters. Between morphology and radiology, only nodular forms were discordant with concordance for diffuse goiter. The correlation between clinicoradiological and clinico-morphological goitrous forms for HT is displayed in Table 5. In HT, there was discordance for ULH and nodular forms in both clinicomorphologic and clinicoradiological correlations as suggested by statistical significance. The discordance in goitrous forms was even noted, between uncut and bisected specimen on macroscopic ex vivo examination, though it was concordant in most of the cases (Fig. 1). This was highlighted by finding nodules with distinct demarcation from surrounding parenchyma on cut section, in clinically, radiologically and intraoperatively diffuse goiters (Fig. 2). This discordance was noted in 9 and 8 cases of GD and HT respectively. Similarly, discordance of diffuse nature was noted on bisected macroscopic examination of clinically nodular goiters (Fig. 3) in 13 cases of GD and HT each. The mean weight of ex vivo goiter in GD and HT was 37.4 \pm 3.2 and $43.3 \pm 4.9 \mathrm{gm}$ respectively. Three cases of HT had papillary microcarcinoma and none of the cases in GD had incidental malignancy. Representative images of atrophic and unilobar hyperplasia are shown in Fig. 4.

\section{DISCUSSION}

Amongst the various thyroid disorders, GD and $\mathrm{HT}$ are two very common conditions, Worldwide. ${ }^{1-5}$ Both of them are organ specific AITD, with a whole gamut of associated autoimmune dermatologic, ${ }^{13}$ gastrointestinal,,${ }^{14}$ hematologic, ${ }^{15}$ endocrinological associations. ${ }^{3,16}$ The classical presentation of GD is rapid onset of severe hyperthyroidism with diffuse goiter and often associated with ophthalmopathy,

Table 3: Frequency distribution of clinical, morphologic and radiologic goiter forms of autoimmune thyroid disease

\begin{tabular}{|c|c|c|c|c|}
\hline \multirow[t]{2}{*}{ Morphology of goiter } & \multicolumn{2}{|c|}{ Graves' disease } & \multicolumn{2}{|c|}{ Hashimoto's thyroiditis* } \\
\hline & Clinical & Radiological & Clinical & Radiological \\
\hline $\begin{array}{l}\text { Unilobar hyperplasia (ULH) } \\
(11)(8)^{*}\end{array}$ & 7 & 9 & 3 & 5 \\
\hline Nodular (5) $(9)^{*}$ & 18 & 8 & 13 & 14 \\
\hline Diffuse (32) $(18)^{*}$ & 23 & 31 & 19 & 16 \\
\hline Atrophic (4) & 4 & 4 & - & - \\
\hline $\begin{array}{l}\text { Marine lenhart syndrome (MLS) } \\
\text { (1) }\end{array}$ & 1 & 1 & - & - \\
\hline
\end{tabular}

*number of cases pertaining to Hashimoto's thyroiditis 
Table 4: Clinicoradiological and clinicomorphological correlation in Grave's disease

\begin{tabular}{lll}
\hline Morphology & Clinical frequency & $\begin{array}{l}\text { Radiological } \\
\text { frequency }\end{array}$ \\
\hline $\begin{array}{l}\text { Unilobar } \\
\text { hyperplasia (ULH) }\end{array}$ & $7(p$-value $=0.09)$ & $9(p$-value $=0.108)$ \\
$(11)$ & & \\
Nodular (5) & $18(p$-value $=0.01)$ & $8(p$-value $=0.07)$ \\
Diffuse (32) & $23(p$-value $=0.04)$ & $31(p$-value $=0.128)$ \\
Atrophic $(4)$ & 4 NA & $4 \mathrm{NA}$ \\
$\begin{array}{l}\text { Marine-Lenhart } \\
\text { syndrome }(M L S)\end{array}$ & $1 \mathrm{NA}$ & $1 \mathrm{NA}$ \\
$(1)$ & & \\
\hline
\end{tabular}

Chi-square and Fisher exact tests were employed; NA: Not applicable

Table 5: Clinicoradiological and clinicomorphological correlation in Hashimoto's thyroiditis

\begin{tabular}{lll}
\hline Morphology & Clinical frequency & Radiological frequency \\
\hline $\begin{array}{l}\text { Unilobar } \\
\text { hyperplasia }\end{array}$ & $3(p$-value $=0.132)$ & $5(p$-value $=0.108)$ \\
$\begin{array}{lll}\text { (ULH) }(8) \\
\text { Nodular }(9)\end{array}$ & $22(p$-value $=0.02)$ & $14(p$-value $=0.04)$ \\
Diffuse $(18)$ & $10(p$-value $=0.01)$ & $16(p$-value $=0.153)$ \\
\hline
\end{tabular}

Chi-square and Fisher exact tests were employed

proximal myopathy and rarely dermatoacropathy, usually in women between 20 and 40 years. Autoimmunity induced by thyrotropin receptor stimulating autoantibodies have been implicated in its causation. ${ }^{17}$ The classical clinical picture of HT is repeated attacks of mild or subclinical inflammation of thyroid gland manifested by subclinical or mild hyperthyroidism followed by a end stage permanent hypothyroidism and a variably sized diffuse, firm goiter, usually in women between 30 and 50 years. ${ }^{7,18}$ But, there are several atypical clinical presentations, which depends on phase of disease, age of the patient, extent of autoimmunity, severity of disease, genetics, geography, etc. Moreover, GD and HT are often confused for one another in 10 to $20 \%$ of cases due to overlapping clinical features and similar natural histories. ${ }^{19,20}$ For instance, GD may present with milder hyperthyroidism in extremes of age groups, ${ }^{21}$ apathetic hyperthyroidism in elderly ${ }^{22,23}$ or as an atrophic goiter. ${ }^{24}$ HT can present with severe thyrotoxicosis, i.e. Hashitoxicosis ${ }^{25}$ or exophthalmos. ${ }^{26,27}$ Often, GD and HT can be co-existent in the same individual. The phenomenon of intermolecular signal shift at genetic/molecular level in $\mathrm{AITD}^{28}$ and clinical progression of GD in to $\mathrm{HT}^{29}$ have been reported, surfacing the debatable hypothesis that GD and HT are spectrum of a same disease process.

One of the important clues in the definitive diagnosis of AITD is the goiter morphology. Both GD and HT usually have diffuse goiter due to bilaterally symmetrical involvement of thyroid gland. But, in 24 to $38 \%$ of cases they may be associated with nodules or asymmetrical enlargement. ${ }^{9}, 10,30$
This leads to diagnostic confusion in cases with atypical clinical picture, especially in nodular goiters. We found very scanty emphasis on the goiter morphology of AITD in the literature. The knowledge of different morphological patterns of goiter can help in accurate diagnosis of AITD. This also ensures adequate counseling of patient regarding outcome, expectations, associated autoimmune conditions and syndromic features like ophthalmopathy, cardiovascular status, etc.

Though GD is known as diffuse toxic goiter, the goiter is not always diffuse. The nature of goiter can be nodular or atrophic. The incidence of nodular goiter was $35 \%$ in this study. Another instance is marine lenhart syndrome (MLS), wherein a solitary toxic nodule may be present within diffuse

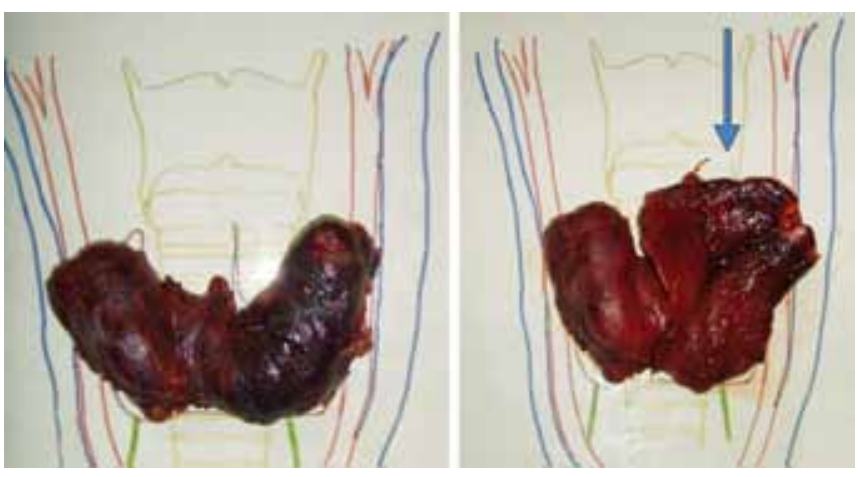

Fig. 1: Concordance between gross uncut (left) and bisected (right) ex vivo specimen

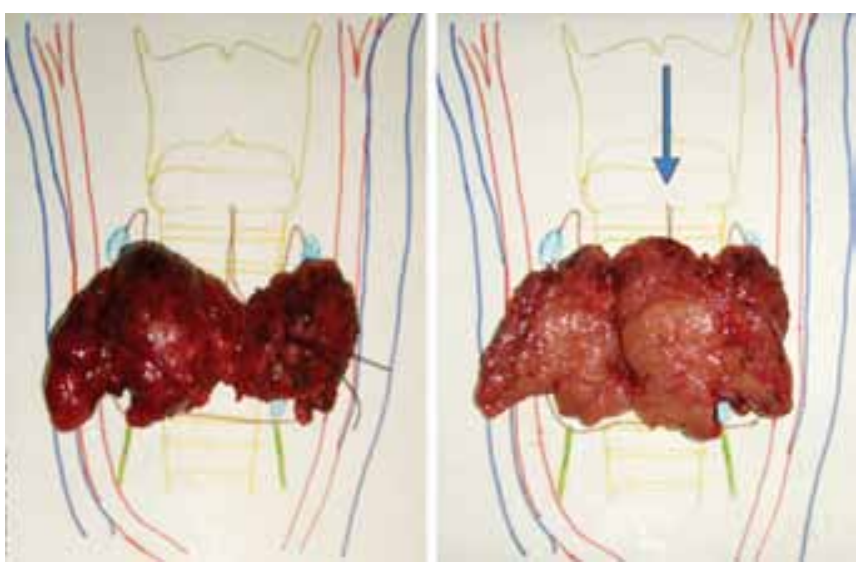

Fig. 2: Discordance between uncut diffuse (left) and nodular (right) bisected specimen

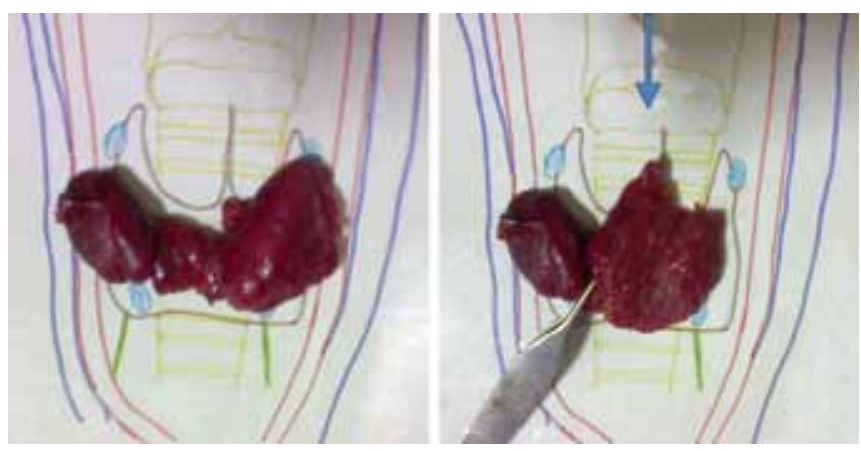

Fig. 3: Discordance between uncut nodular (left) and diffuse (right) bisected specimen 


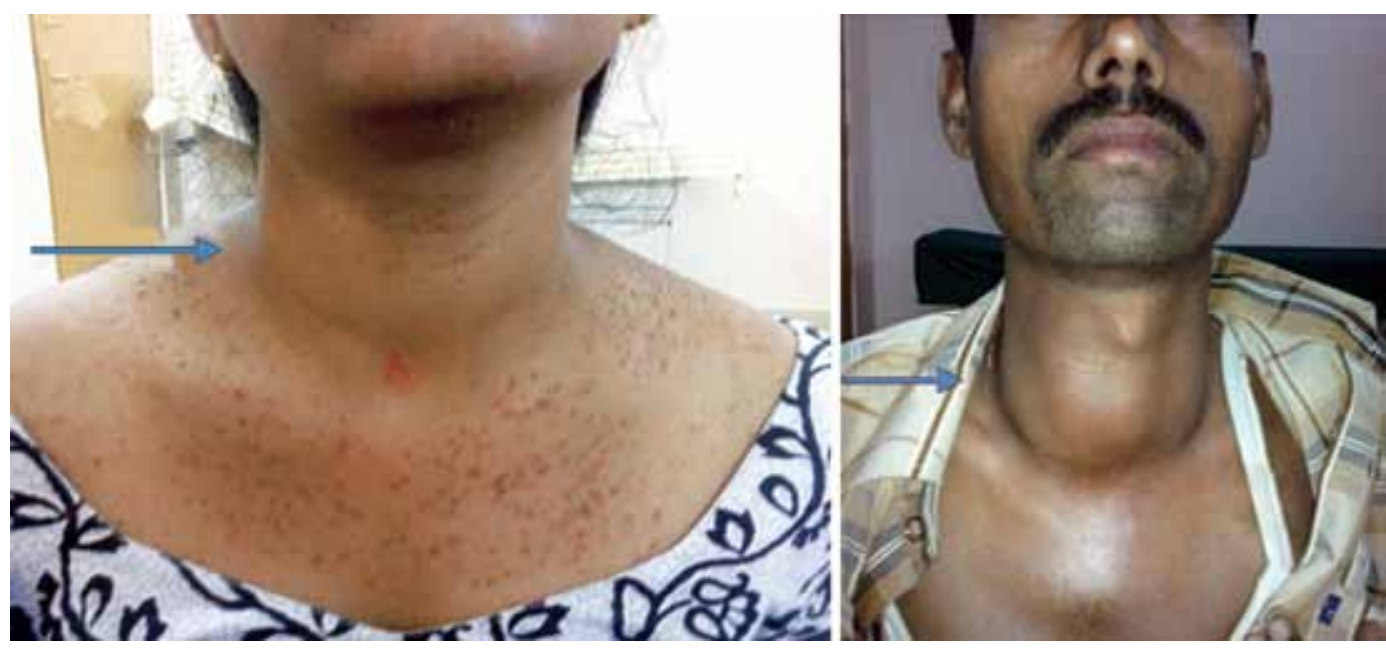

Fig. 4: Graves' disease clinical appearence-Nongoitrous form (left); Unilobar goiter (right)

toxic goiter. ${ }^{31}$ Clinically and radiologically, this springs up differential diagnosis with toxic multinodular goiter (TMNG), Toxic adenoma or acute stages of thyroiditis. Radioisotope scan may not accurately diagnose these atypical cases, as scintiscan and clinical picture are discordant. Histopathology of GD is highly nonspecific, due to lack of stringent diagnostic criteria in contrast to conditions such as papillary thyroid carcinoma, medullary cancer, HT, etc. The routine diagnosis of GD is primarily done on clinical basis substantiated by histopathology in operated cases. But, the heterogenous morphologic forms of goiter in GD are not well documented. In absence of specific cytology and histopathology findings, the diagnosis of GD is often missed or underdiagnosed, as it is confused with TMNG or Toxic adenoma or HT in a surgical series.

Moreover, GD and HT can be co-existent in a goiter histopathology as seen in 6 cases of our series. This is usually seen as islands of HT interspersed between hyperplastic follicles. The overlap with other conditions and nonspecificity of histopathology in GD, is also due to factors, such as stage of disease-active or inactive treated state or burnout phase, reactive hyperplasia, colloid content, autoimmune status, follicular autonomy, degree of hyperplasia and variable clonal stimulation within GD. This nonspecific histopathology of GD is also highlighted in our study by the pathologist's report of GD cases, showing colloid nodule, hyperplastic goiter and adenomatous hyperplasia. It is also known that, there is variability in susceptibility of various follicular cell clones to stimulatory factors within thyroid gland. Multiple genetic factors and HLA status influence thyroid goitrogenesis. ${ }^{32,33}$ This might lead to atypical morphological forms like atrophic goiter, asymmetrical hyperplasia. The intrathyroidal follicular microenvironment is a complex milieu with multiple genetic and environmental factors influencing it. This could lead to variability in morpho- logy, histopathology and natural course of disease. Various theories on goitrogenesis based on dietary iodine status, ${ }^{34}$ molecular, ${ }^{35}$ thyrocyte clonality ${ }^{36}$ emphasize the complex etiology of goitrogenesis in the thyroid gland. Moreover, nodules may take priority over rest of the parenchyma in histopathological evaluation. All the above factors, can lead to misdiagnosis or underdiagnosis of AITD. Follow-up and counseling are thus compromised, especially with the present short stay trends in surgery.

Diffuse goiters were more common in GD compared to HT, as seen in $35 \%$ compared to $23 \%$ respectively, in this study. This is probably due to uniform effect of stimulatory autoantibodies on the entire thyroid parenchyma in GD. In HT, autoimmunity initially causes parenchymal destruction followed by compensatory hyperplasia leading to propensity for nodule formation. Clinically, goiter characterization and differentiation between a true nodule vs pseudonodularity and diagnosis of a nodule is inaccurate, with better accuracy for larger nodules. ${ }^{37}$ To obviate this low sensitivity of clinical palpation, nodularity was confirmed by cut section to differentiate it from pseudonodularity. Sonographically, pseudonodularity or pseudotumors were described as nodular HT as hypoechoic with ill-defined margins. ${ }^{11,38}$ Pseudonodularity can occur due to asymmetrical growth secondary to focal hyperplasia or fibrous septa between hypertrophic areas and surrounding parenchyma, which are confused with true nodularity clinicoradiologically. A true nodule has distinct demarcation with surrounding parenchyma and capsule surrounding it. This is best seen on cut section morphology.

The reason for nodule formation in HT could be, due to variable extent of inflammation within the thyroid gland, i.e. focal thyroiditis and fibrous variant. Nodule formation can occur due to neoplastic component or colloid nodule within the gland with HT or GD. ${ }^{10,39,40}$ Nodular goiter was due to 
follicular adenoma and colloid nodule in two and four HT cases respectively. While, differentiated thyroid cancer can co-exist with GD or HT, it was not included in this study to avoid bias of malignant nodules. Cases with incidental microcarcinoma on histopathology were included as they did not alter goiter morphology.

Pertinent factors in developing countries, such as: (1) Increased prevalence of nodular goiters; (2) frequent inaccessibility to scintigraphy facility; (3) frequent inaccessibility and financial constraints for TSH Ab testing; (4) lack of robust diagnostic criteria on histopathology of GD; (5) false positive and false negative scintiscans due to partial treatment or stage of disease in natural history of AITD and (6) lack of definitive diagnosis before and after surgery in many peripheral medical setups, appears to justify the morphologic (Gross and Cut) evaluation as more objective than clinical or radiological assessement of goiter to ascertain true $v s$ pseudonodularity. But, there is a learning curve for morphological assessment and clinician needs to practice it routinely to include it in his armamentarium for definitive diagnosis of AITD.

\section{CONCLUSION}

1. Autoimmune thyroid disease has heterogenous goitrous forms.

2. Macroscopic morphological evaluation of goiter complements the clinicoradiological-pathological diagnosis of AITD.

3. Knowledge of the morphological variants helps in optimal final diagnosis, management and follow-up of AITD.

\section{REFERENCES}

1. Tunbridge WM, Evered DC, Hall R, Appleton D, Brewis M, Clark F, Evans JG, Young E, Bird T, Smith PA. The spectrum of thyroid disease in a community: the Whickham survey. Clin Endocrinol (Oxf) 1977;7(6):481-493.

2. Cooper DS, Doherty GM, Haugen BR, et al. American thyroid association (ATA) guidelines taskforce on thyroid nodules and differentiated thyroid cancer. Revised American thyroid association management guidelines for patients with thyroid nodules and differentiated thyroid cancer. Thyroid 2009;19(11):1167-1214.

3. Levine SN. Current concepts of thyroiditis. Arch Intern Med 1983;143(10):1952-1956.

4. Kochupillai N. Clinical endocrinology in India. Current Science 2000;79(8):1061-1067.

5. Vanderpump MP, Tunbridge WM. Epidemiology and prevention of clinical and subclinical hypothyroidism. Thyroid 2002;12(10): 839-847.

6. Wiebolt J, Achterbergh R, den Boer A, van der Leij S, Marsch E, Suelmann B, et al. Clustering of additional autoimmunity behaves differently in Hashimoto's patients compared with Graves' patients. Eur J Endocrinol 2011;164(5):789-794.

7. Pearce EN, Farwell AP, Braverman LE. Thyroiditis. N Engl J Med 2003;348(26):2646-2655.

8. Dobyns BM, Sheline GE, Workman JB, Tompkins EA, McConahey WM, Becker DV. Malignant and benign neoplasms of the thyroid in patients treated for hyperthyroidism: a report of the cooperative thyrotoxicosis therapy follow-up study. J Clin Endocrinol Metab 1974;38(6):976-998.

9. Cantalamessa L, Baldini M, Orsatti A, Meroni L, Amodei V, Castagnone D. Thyroid nodules in graves disease and the risk of thyroid carcinoma. Arch Intern Med 1999;159(9):1705-1708.

10. Pradeep PV, Agarwal A, Baxi M, Agarwal G, Gupta SK, Mishra SK. Safety and efficacy of surgical management of hyperthyroidism: 15-year experience from a tertiary care center in a developing country. World J Surg 2007 Feb; 31(2):306-312.

11. Takashima S, Matsuzuka F, Nagareda T, Tomiyama N, Kozuka T. Thyroid nodules associated with Hashimoto's thyroiditis: assessment with US. Radiology 1992;185(1):125-130.

12. World Medical Organization. Declaration of Helsinki. BMJ 1996; 313:1448-1449.

13. Heymann WR. Cutaneous manifestations of thyroid disease. J Am Acad Dermatol 1992;26(6):885.

14. Doniach D, Roitt IM, Taylor KP. Autoimmune phenomena in periniocious anemia: serological overlap with thyroiditis, thyrotoxicosis, and systemic lupus erythematosus. BMJ 1963;1(5342): 1374-1379.

15. Hymes K, Blum M, Lackner H, et al. Easy bruising, thyrombocytopenia, and elevated platelet immunoglobulin $\mathrm{G}$ in Graves' disease and Hashimoto's thyroiditis. Ann Intern Med 1981;94(1):27.

16. Boelaert K, Newby PR, Simmonds MJ, et al. Prevalence and relative risk of other autoimmune diseases in subjects with autoimmune thyroid disease. Am J Med 2010;123(2):183.

17. Rapoport B, Chazenbalk GD, Jaume JC, McLachlan SM. The thyrotropin receptor: Interaction with thyrotropin and autoantibodies. Endocr Rev 1998;19(6):673-716.

18. Sakiyama R. Thyroiditis: a clinical review. Am Fam Physician 1993;48(4):615-621.

19. Falk SA, Birken EA, Ronquillo A. Graves' disease associated with histologic Hashioto's thyroiditis. Otolaryngol Head Neck Surg 1985;93(1):86-91.

20. Wood LC, Ingbar SH. Hypothyroidism as a late sequela in patient with Graves' disease treated with antithyroid agents. J Clin Invest 1979;64(5);1429-1436.

21. Grewal RP. Apathetic hyperthyroidism in an adolescent. J Psychiatry Neurosci 1993;18(5):276.

22. Kennedy JW, Caro JF. The ABCs of managing hyperthyroidism in the older patient. Geriatrics 1996;51(5):22-24.

23. Trivalle C, Doucet J, Chassagne P, Landrin I, Kadri N, Menard JF, Bercoff E. Differences in the signs and symptoms of hyperthyroidism in older and younger patients. J Am Geriatr Soc 1996;44(1):50-53.

24. Laurberg P, Buchholtz Hansen PE, Iversen E, Eskjaer Jensen S, Weeke J. Goitre size and outcome of medical treatment of Graves' disease. Acta Endocrinol (Copenh) 1986;111(1):39-43.

25. Nabhan ZM, Kreher NC, Eugster EA, Hashitoxicosis in children: clinical features and natural history. J Pediatr 2005;146(4):533536.

26. Shirakawa H, Amemiya T, Kasagi K, et al. Hashimoto disease with hyperthyroid ocular signs. Metab Pediat Ophthalmol 1981;5(3-4): 213-218.

27. Bartalena L, Pinchera A, Marcocci C. Management of Graves' ophthalmopathy: reality and perspetives. Endocrine Rev 2000; 21(2):168-199.

28. McLachlan SM, Nagayama Y, Pichurin PN, Mizutori Y, Chen CR, Misharin A, et al. The Link between Graves' disease and Hashimoto's thyroiditis: a role for regulatory. T Cells Endocrinology 2007;148(12):5724-5733. 
29. Umar H, Muallima N, Adam JM, Sanusi H. Hashimoto's thyroiditis following Graves' disease. Acta Med Indones 2010;42(1): 31-35.

30. Isik S, Gokay F, Ozuguz U, Topaloglu O, Tutuncu Y, Berker D, Guler S. Comparison of the prevalence and sonographic features of thyroid nodules accompanying autoimmune thyroid diseases. Endokrynol Pol 2010;61(6):658-664.

31. Biersack HJ, Biermann K. The Marine-Lenhart syndrome revisited. Wien Klin Wochenschr 2011;123(15-16):459-462.

32. Stenszky V, Kozma L, Balazs C. The genetics of Graves' disease. HLA and disease susceptilbility. J Clin Endocrinol Metab 1985; 61(4):735-740.

33. Blackmore AIF, et al. Association of Graves' disease with an allele of the interleukin-I receptor antagonist gene. J Clin Endocrinol Metab 1995;80(1):111-115.

34. Marine D, Kimball OP. The prevention of simple goiter in man. J Lab Clin Med 1917;3:40-48.
35. Krohn K, Führer D, Holzapfel HP, Paschke R. Clonal origin of toxic thyroid nodules with constitutively activating thyrotropin receptor mutations. J Clin Endocrinol Metab 1998;83(1):130-134.

36. Derwahl M, Studer H. Nodular goiter and goiter nodules: where iodine deficiency falls short of explaining the facts. Exp Clin Endocrinol Diabetes 2001;109(5):250-260.

37. Tan GH, Gharib H, Carl C. Reading, MD solitary thyroid nodule comparison between palpation and ultrasonography. Arch Intern Med 1995;155(22):2418-2423.

38. Langer JE, Khan A, Nisenbaum HL, et al. Sonographic appearance of focal thyroiditis. AJR 2001;176(3):751-754.

39. Bhargav PR, Shekhar S. Surgical indications for goiter with background Hashimoto's thyroiditis: Institutional experience. Indian J Surg 2011;73(6):414-418.

40. Carnell NE, Valente WA. Thyroid nodules in Graves' disease: classification, characterization, and response to treatment. Thyroid 1998;8(8):647-652. 